\title{
Co-culture of hepatocellular carcinoma cells and human umbilical endothelial cells damaged by SU11274
}

\author{
MINORU TOMIZAWA ${ }^{1}$, FUMINOBU SHINOZAKI ${ }^{2}$, YASUFUMI MOTOYOSHI ${ }^{3}$, \\ TAKAO SUGIYAMA $^{4}$, SHIGENORI YAMAMOTO ${ }^{5}$ and NAOKI ISHIGE ${ }^{6}$ \\ Departments of ${ }^{1}$ Gastroenterology, ${ }^{2}$ Radiology, ${ }^{3}$ Neurology, ${ }^{4}$ Rheumatology, ${ }^{5}$ Pediatrics and ${ }^{6}$ Neurosurgery, \\ National Hospital Organization Shimoshizu Hospital, Yotsukaido, Chiba 284-0003, Japan
}

Received July 30, 2014; Accepted September 2, 2014

DOI: 10.3892/br.2014.361

\begin{abstract}
Mesenchymal-epithelial transition factor (c-Met) is a receptor that binds to the hepatocyte growth factor and is upregulated in hepatocellular carcinoma (HCC). The anti-tumor effects of (3Z)-N-(3-chlorophenyl)-3-(\{3,5-dimethyl-4-[(4methyl-piperazin-1-yl)carbonyl]-1H-pyrrol-2-yl\}methylene)-N-methyl-2-oxo-2,3-dihydro-1H-indole-5-sulfonamide (SU11274), a c-Met inhibitor, were investigated in the present study. HCC cells (HLE, HLF, PLC/PRL/5, Hep3B, Huh-6 and HepG2) and human umbilical vein endothelial cells (HUVECs) were used. Quantitative polymerase chain reaction was performed to detect the expression level of c-Met in HCC and HUVECs, and cyclin D1 in HCC. The 3-(4,5-dimethylthiazol-2-yl)-5-(3-carboxymethoxyphenyl)-2-(4-sulfophenyl)-2H-tetrazolium inner salt assay was performed to assess the proliferation of the HCC cells and HUVECs cultured with SU11274. Co-culture of HLF or PLC/PRL/5 cells and HUVECs was established as an in vitro model of HCC tissues. The expression levels of c-Met in HLE, HLF, PLC/PRL/5, Hep3B, Huh-6 and HepG2, adult healthy liver and HUVECs were $4.43 \pm 0.50,1.61 \pm 0.18$, $3.70 \pm 0.08,0.81 \pm 0.18,6.60 \pm 1.29,1.06 \pm 0.35,1.00 \pm 0.09$ and $88.8 \pm 17.3$ (mean \pm standard deviation), respectively. SU11274 (30 $\mu \mathrm{M})$ suppressed the proliferation of HLF, PLC/PRL/5 and HUVECs to $11.0 \pm 9.4,46.5 \pm 30.7$ and $29.4 \pm 5.0 \%$, respectively. SU11274 $(30 \mu \mathrm{M})$ decreased the expression levels of cyclin D1 in HLF and PLC/PRL/5 cells to $45.1 \pm 11.6$ and $30.1 \pm 10.3 \%$, respectively. SU11274, at a concentration of $30 \mu \mathrm{M}$ damaged the morphology of the co-cultures of HLF or PLC/PRL/5 cells with HUVECs and all the cells died.c-Met is highly expressed in HUVECs and HCC cells, but not in Hep3B. At a 30- $\mu \mathrm{M}$ concentration, SU11274 suppresses the proliferation of HLF, PLC/PRL/5 and HUVECs. SU11274 $(30 \mu \mathrm{M})$ damages the co-cultures of HLF or PLC/PRL/5 cells with HUVECs.
\end{abstract}

Correspondence to: Dr Minoru Tomizawa, Department of Gastroenterology, National Hospital Organization Shimoshizu Hospital, 934-5 Shikawatashi Street, Yotsukaido, Chiba 284-0003, Japan

E-mail: nihminor-cib@umin.ac.jp

Key words: mesenchymal-epithelial transition factor, quantitative polymerase reaction

\section{Introduction}

Hepatocellular carcinoma (HCC) is a common malignancy of the liver with a poor prognosis. HCC is treated with local ablation, surgical resection, transcatheter arterial chemoembolization and systemic administration of chemotherapeutic agents $(1,2)$. The HCC tissue obtains nutrients and oxygen from blood vessels by the process of angiogenesis $(3,4)$. Vascular endothelial cells proliferate by activating the mitogen-activated protein (MAP) kinase pathway, which acts downstream of the vascular endothelial cell growth factor (VEGF) receptor (5). Sorafenib, a multikinase inhibitor of VEGF, inhibits the MAP kinase pathway and is used to treat HCC $(6,7)$. Despite the sorafenib treatment, $>25 \%$ of patients with HCC succumb from disease progression (8). Thus, it is necessary to investigate alternative drugs/reagents alone or in combination with sorafenib that can inhibit the signaling pathways involved in the proliferation of HCC.

Mesenchymal-epithelial transition factor (c-Met) is a receptor of the hepatocyte growth factor (HGF) (9). When HGF binds to c-Met, it stimulates downstream signaling via the phosphatidylinositol-3 kinase pathway and MAP kinase. Since c-Met is upregulated in HCC tissues compared to the surrounding normal tissues $(10,11)$, inhibitors of c-Met, including (3Z)-N-(3-chlorophenyl)3-(\{3,5-dimethyl-4-[(4-methylpiperazin-1-yl)carbonyl]-1H-pyrrol -2-yl\}methylene)-N-methyl-2-oxo-2,3-dihydro-1H-indole-5-sulf onamide (SU11274), are considered to be promising candidates for the treatment of HCC (12). SU11274 competes with adenosine triphosphate to bind to the activation loop of c-Met (13). SU11274 is known to suppress the proliferation of HCC cells (14), and if SU11274 can also suppress angiogenesis, the anti-tumor effects are expected to be stronger.

Thus, the aim of the present study was to establish a co-culture of HCC and human umbilical vein endothelial cells (HUVECs) as an in vitro model of the HCC tissue and to investigate the anti-tumor effects of SU11274.

\section{Materials and methods}

Ethical statement. The study was approved by the Ethics Committee of National Hospital Organization Shimoshizu Hospital (Yotsukaido, Japan). Human fetal and adult liver RNA were collected with informed consent from the donors 
and their relatives by Clontech Laboratories, Inc., (Mountain View, CA, USA). All the human tissue samples were collected with informed consent from the donors and their relatives by BioChain (Hayward, CA, USA).

Cell culture. HCC cell lines (HLE, HLF, PLC/PRL/5, Hep3B and HepG2) were purchased from RIKEN Cell Bank (Tsukuba, Japan). The cells were cultured in Dulbecco's modified Eagle's medium (DMEM; Sigma-Aldrich, St. Louis, MO, USA) and supplemented with $10 \%$ fetal bovine serum (FBS; Life Technologies, Grand Island, NY, USA). HUVECs and their culture medium [endothelial cell growth media bullet kit (EGM)] were purchased from Lonza (Walkersville, NJ, USA). The cell lines were cultured in $5 \%$ carbon dioxide at $37^{\circ} \mathrm{C}$ in a humidified chamber. The cultured cells were observed under a microscope (CKX41N-31PHP; Olympus, Tokyo, Japan).

Hematoxylin and eosin $(H \& E)$ staining. The cells were spread onto 4-well chambers (Beckton Dickinson, Franklin Lakes, NJ, USA), fixed with $100 \%$ methanol at room temperature and stained with H\&E staining (Muto Pure Chemicals Co., Ltd., Tokyo, Japan). The specimens were observed and images were captured using an AX80 microscope (Olympus).

Co-culture of HLF or PLC/PRL/5 cells with HUVECs. HUVECs were spread at a density of $1.9 \times 10^{4}$ cells onto each well of a 24-well plate coated with matrigel (Becton Dickinson) at the density of each well. After 1-day culture in EGM, the medium was discarded and 1.9 $\times 10^{4} \mathrm{HLF}$ cells or PLC/PRL/5 cells were spread onto each well. The cells were cultured in DMEM supplemented with 10\% FBS.

Immunostaining. Serial sections of human healthy adult liver (64-year-old male) and HCC tissue (60-year-old female) (BioChain) were deparaffinized, autoclaved and incubated with hydrogen peroxide, followed by $2 \%$ FBS in phosphate-buffered saline (PBS; washing buffer) for $30 \mathrm{~min}$. Following an overnight incubation with rabbit monoclonal anti-c-Met antibody (1:300; Cell Signaling Technology, Danvers, MA, USA), the specimens were rinsed with PBS and incubated with horseradish peroxidase-labeled anti-rabbit antibody $(1: 2,000$; GE Healthcare, Pittsburgh, PA, USA) for $2 \mathrm{~h}$. Subsequently, diaminobenzidine (Dako, Glostrup, Denmark) was applied to the tissue sections as a chromogen and the nuclei were stained with hematoxylin for $15 \mathrm{sec}$. The specimens were observed and the images were captured under an AX80 microscope (Olympus). A specimen of HCC tissue incubated without the primary antibody was used as a negative control.

Cell proliferation analysis. HLF cells, PLC/PRL/5 cells or HUVECs were trypsinized, harvested, spread onto 96-well flat-bottom plates (Asahi Techno Glass, Tokyo, Japan) at a density of 1,000 cells per well and were incubated for $24 \mathrm{~h}$ in media supplemented with $10 \%$ FBS. The cells were treated with the c-Met inhibitor, SU11274 (Wako Pure Chemicals, Tokyo, Japan) at $0,0.03,0.1,0.3,1,3,10$ and $30 \mu \mathrm{M}$ for $72 \mathrm{~h}$. These cells were subsequently used in the 3-(4,5-dimethylthiazol-2-yl)-5(3-carboxymethoxyphenyl)-2-(4-sulfophenyl)-2H-tetrazolium, inner salt (MTS) assays, according to the manufacturer's instructions (Promega Corporation, Madison, WI, USA). MTS is bioreduced by the cells into a colored formazan product into a colored formazan product. The absorbance was analyzed at a wavelength of $490 \mathrm{~nm}$ with an iMark Microplate Absorbance Reader (Bio-Rad, Hercules, CA, USA). The absorbance was normalized against that of $0 \mu \mathrm{M}$.

Reverse transcription quantitative polymerase chain reaction $(R T-q P C R)$. The cells were spread in 6-well plates (Asahi Techno Glass) and cultured until they reached $80 \%$ confluency. SU11274 was added to the media and $48 \mathrm{~h}$ later, total RNA was isolated using a kit (Isogen; Nippon Gene, Tokyo, Japan). Complimentary DNA was synthesized using SuperScript III and oligo(dT) primers (Life Technologies), as per the manufacturer's instructions. Total RNA from adult human healthy liver was purchased from Clontech Laboratories, Inc. The PCR primers and product sizes were as follows: c-Met 77 [NM_000245; 5'-CATTGGGGAGCACTATGTC-3' (forward), 5'-TGT 78 CCACCTCATCATCAGCG-3' (reverse); 110 basepairs (bp)], cyclin D1 (NM_053056; 5'-AGAGGCGGAGGA GAACAAACAG-3', 5'-AGGCGGTAGTAGGACAGGAAG TTG-3'; 180 bp) and RPL19 (BC095445; 5'-CGAATGCCAG AGAAGGTCAC-3', 5'-CCATGAGAATCCGCTTGTTT-3'; $157 \mathrm{bp}$ ). RT-qPCR was performed at 40 cycles consisting of denaturing for $5 \mathrm{sec}$ and annealing-extension for $5 \mathrm{sec}$. RPL19 primers were used as the internal controls. RT-qPCR was performed using the Fast SYBR-Green Master mix (Life Technologies) in the MiniOpticon system (Bio-Rad). The expression level of the genes was analyzed automatically with the system. The expression levels were normalized against that of $0 \mu \mathrm{M}$.

Statistical analyses. One-way analysis of variance was performed with JMP 10.0.2 (SAS Institute, Cary, NC, USA). $\mathrm{P}<0.05$ was considered to indicate a statistically significant difference.

\section{Results}

c-Met expression. The expression of c-Met was analyzed immunohistochemically in 99 surgical specimens of adult healthy liver and HCC samples from 100 that were commercially available. In the absence of the anti-c-Met antibody, HCC tissues did not show any staining (Fig. 1A). While the cell membrane of hepatocytes in the normal liver was weakly positive for c-Met (Fig. 1B), the cell membrane and cytoplasm of cells in the HCC tissue were positive (Fig. 1C). These data indicate that c-Met is upregulated in HCC cells as compared to the normal hepatocytes.

Subsequently, RT-qPCR was performed to assess the expression level of c-Met in the HCC cell lines and HUVECs (Fig. 2). The expression levels of c-Met in HLE, HLF, PLC/PRL/5, Hep3B, Huh-6, HepG2, adult healthy liver and HUVECs were $4.43 \pm 0.50,1.61 \pm 0.18,3.70 \pm 0.08,0.81 \pm 0.18$, $6.60 \pm 1.29,1.06 \pm 0.35,1.00 \pm 0.09$ and $88.8 \pm 17.3$ (mean \pm standard deviation), respectively. Except in Hep3B cells, the expression of c-Met was higher in all the HCC cell lines and HUVECs as compared to the adult healthy liver.

c-Met inhibition by SU11274. To address the possibility that inhibition of c-Met suppresses cell proliferation, SU11274 

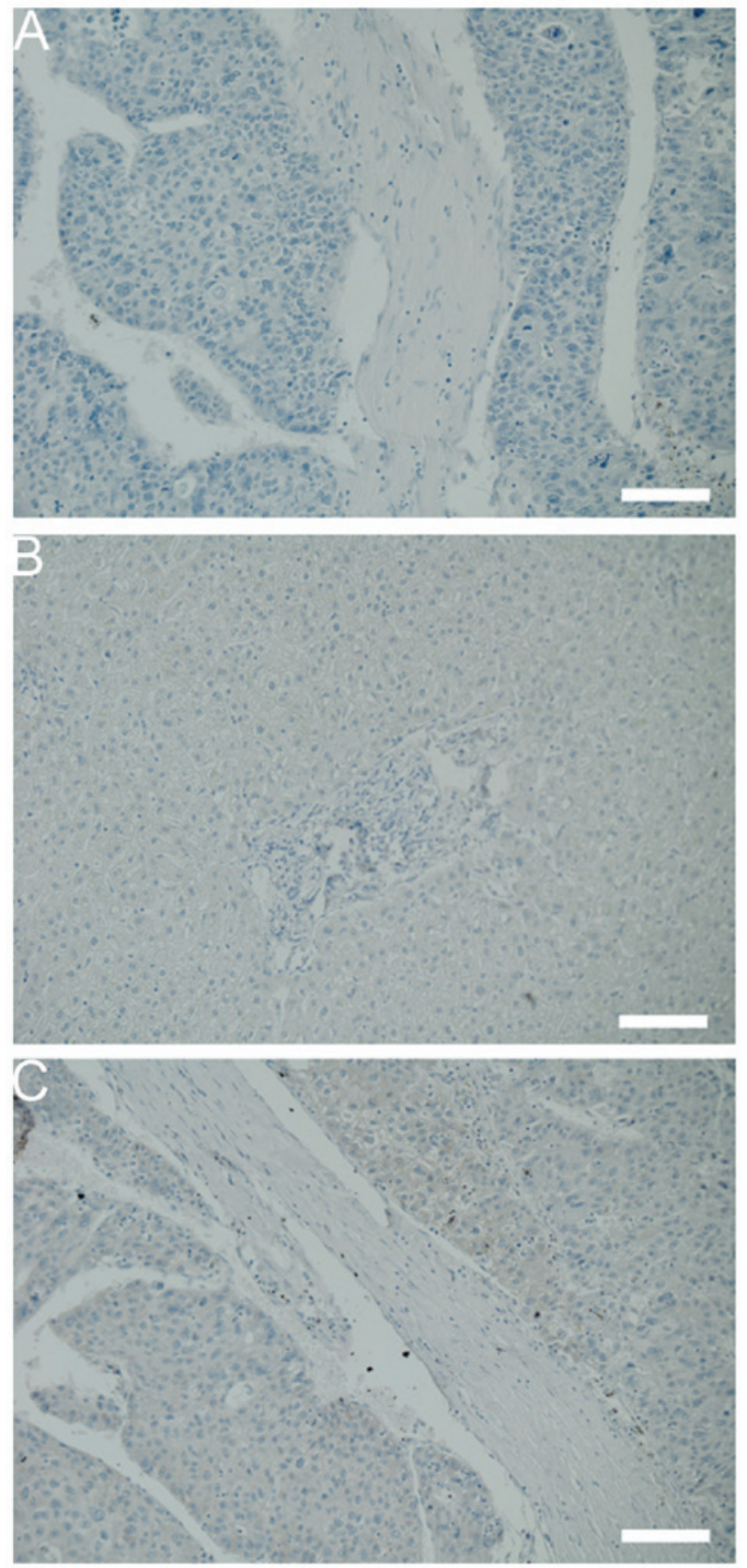

Figure 1. Immunostaining with anti-mesenchymal-epithelial transition factor (c-Met) antibody. Surgical specimens were immunostained with anti-c-MET antibody. (A) A section of hepatocellular carcinoma tissue incubated without the primary antibody was used as the negative control. (B) Normal hepatocytes are weakly positive for c-Met. (C) Hepatocellular carcinoma tissue stains positive for c-Met. Original magnification, x100; scale bar, $100 \mu \mathrm{m}$.

was added to the media and the MTS assay was performed. SU11274 was found to suppress the proliferation of HLF cells in a dose-dependent manner and reached $11.0 \pm 9.4 \%(\mathrm{P}<0.05)$ with $30 \mu \mathrm{M}$ SU11274 as compared to $0 \mu \mathrm{M}$ (Fig. 3A). SU11274 $(30 \mu \mathrm{M})$ suppressed the proliferation of PLC/PRL/5 cells (Fig. 3B) and HUVECs (Fig. 3C) to 46.5 $\pm 30.7(\mathrm{P}<0.05)$ and $29.4 \pm 5.0 \%$, as compared to $0 \mu \mathrm{M}(\mathrm{P}<0.05)$, respectively.

To investigate the mechanism of cell proliferation suppression by SU11274, the expression level of cyclin D1 was analyzed by RT-qPCR and was found to decrease to $45.1 \pm 11.6 \%(\mathrm{P}<0.05)$ with $30 \mu \mathrm{M}$ SU11274 in HLF cells, as

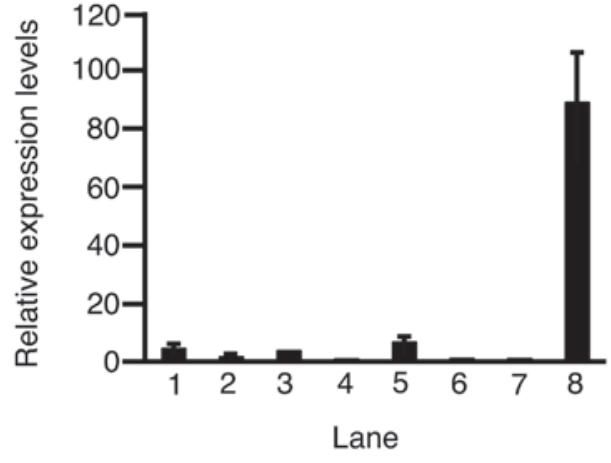

Figure 2. Expression level of mesenchymal-epithelial transition factor (c-Met) in hepatoma cell lines and human umbilical vein endothelial cells (HUVECs). Reverse transcription quantitative polymerase chain reaction was performed to analyze the expression level of c-Met in hepatocellular carcinoma cell lines and HUVECs and compared to healthy adult human liver. The expression level (lanes 1-6 and 8) was normalized against adult human healthy liver (lane 7). Lane 1, HLE; lane 2, HLF; lane 3, PLC/PRL/5; lane 4, Hep3B; lane 5, Huh-6; lane 6, HepG2; lane 7, adult liver; and lane 8, HUVECs; $\mathrm{n}=3$.

A

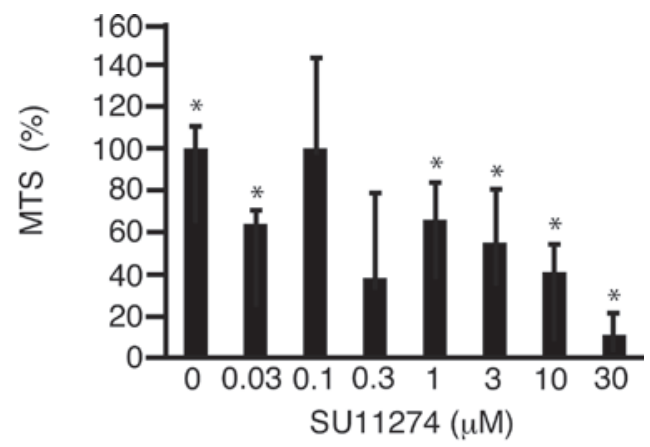

B

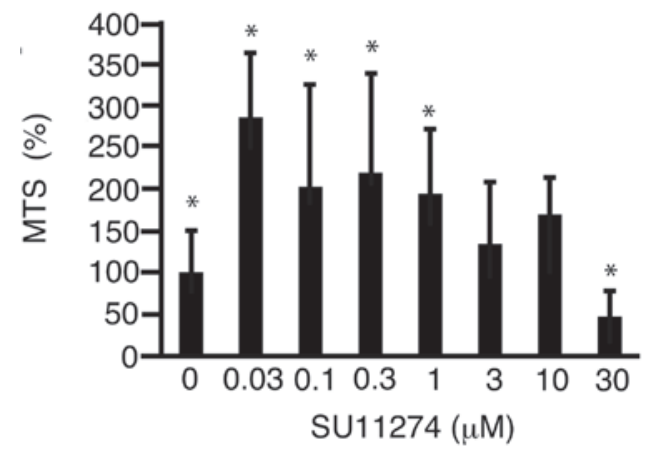

C

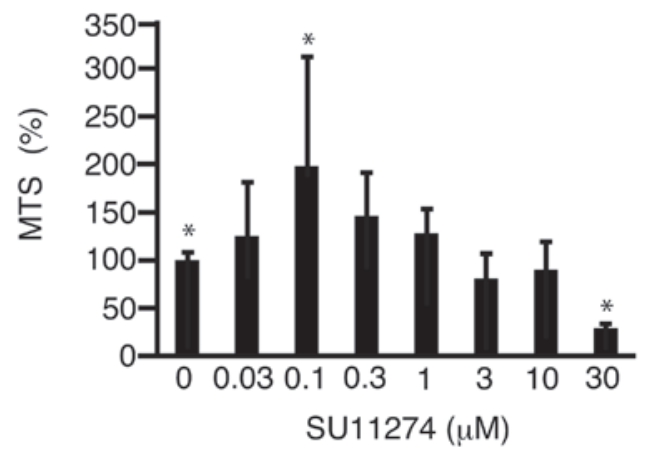

Figure 3. Assay for the proliferation of HLF, PLC/PRL/5 and human umbilical vein endothelial cells (HUVECs). HLF, PLC/PRL/5 and HUVECs were cultured with SU11274. The 3-(4,5-dimethylthiazol-2-yl)-5-(3-carboxymetho xyphenyl)-2-(4-sulfophenyl)-2H-Tetrazolium, inner salt assay was performed with (A) HLF cells, (B) PLC/PRL/5 cells and (C) HUVECs $72 \mathrm{~h}$ after the addition of SU11274. The absorbance at $490 \mathrm{~nm}$ was normalized against that of $0 \mu \mathrm{M}$. ${ }^{*} \mathrm{P}<0.05 ; \mathrm{n}=3$. 
A

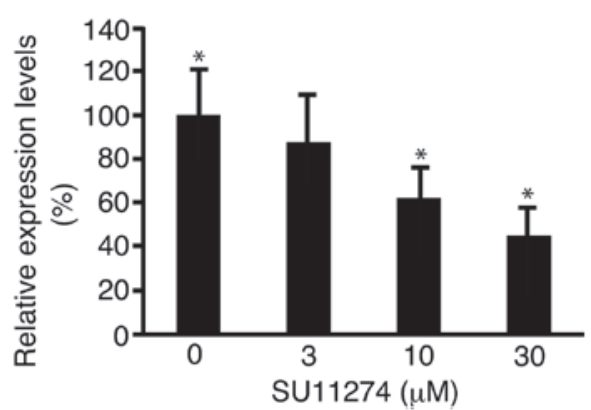

B

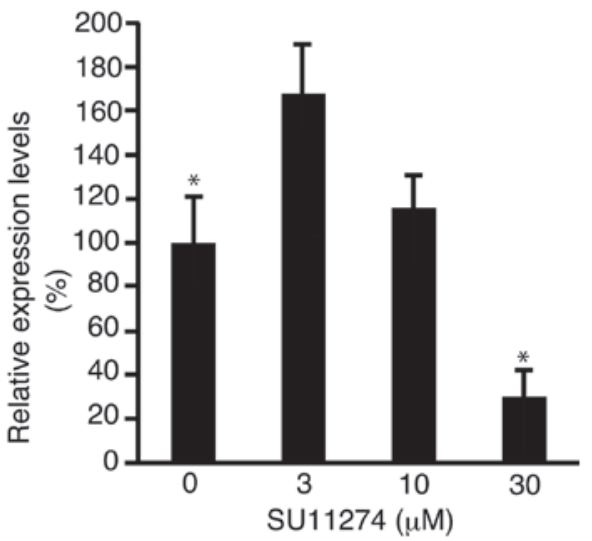

Figure 4. Expression levels of cyclin D1. Reverse transcription quantitative polymerase chain reaction was performed to analyze the expression level of cyclin D1 in (A) HLF cells and (B) PLC/PRL/5 cells. The expression levels were normalized against that of $0 \mu \mathrm{M}$. "P<0.05; $\mathrm{n}=3$.
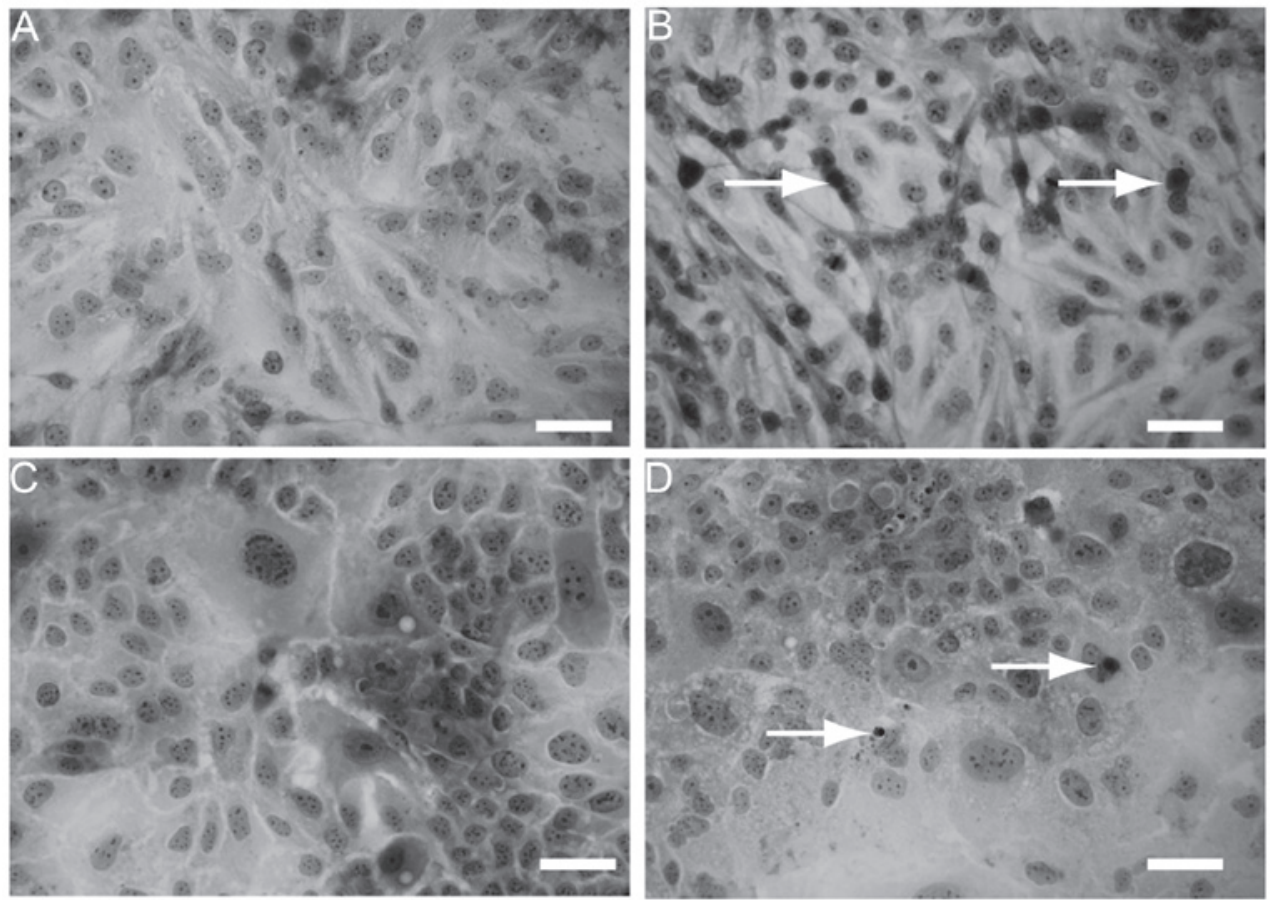

Figure 5. Hematoxylin and eosin (H\&E) staining. HLF and PLC/PRL/5 cells were cultured with and without SU11274 (30 $\mu \mathrm{M}$ ) for $48 \mathrm{~h}$ and subjected to H\&E staining. Apoptotic cells showing pyknotic nuclei (arrow) appeared with SU11274 treatment. (A) HLF cells without SU11274, (B) HLF cells with $30 \mu$ M SU11274, (C) PLC/PRL/5 cells without SU11274 and (D) PLC/PRL/5 cells with $30 \mu \mathrm{M}$ SU11274.

compared to $0 \mu \mathrm{M}$ (Fig. 4A). The expression level of cyclin D1 decreased to $30.1 \pm 10.3 \%(\mathrm{P}<0.05)$ of SU11274 in PLC/PRL/5 cells, as compared to $0 \mu \mathrm{M}$ (Fig. 4B).

In addition, H\&E staining was performed to observe the morphological changes of the cells cultured with or without SU11274. Compared to HLF (Fig. 5A) and PLC/PRL/5 cells (Fig. 5C) without SU11274 treatment, apoptotic cells were observed with $30 \mu \mathrm{M}$ SU11274 treatment (Fig. 5B and D).

Co-cultures of HLF (Fig. 6A) or PLC/PRL/5 cells (Fig. 6C) with HUVECs were established to analyze the possibility that SU11274 damages the co-culture used as an in vitro model of hepatoma tissues. Three days after the addition of $30 \mu \mathrm{M}$ SU11274, the morphology of the co-cultures of HLF (Fig. 6B) or PLC/PRL/5 cells (Fig. 6D) with HUVECs were found to be damaged and as a result, all the cells died.

\section{Discussion}

SU11274 suppresses proliferation and induces apoptosis of HCC cells (15). In the present study, proliferation of HCC cells was suppressed via the downregulation of cyclin D1, a protein involved in cell cycle progression $(16,17)$. These results indicate that SU11274 can suppress cell cycle progression. In addition, H\&E staining showed apoptosis in cells cultured with SU11274. SU11274 is known to activate caspase-3 (15). Taken together, these data indicate that SU11274 suppresses the proliferation of HLF and PLC cells by downregulating cyclin D1 and inducing apoptosis.

c-Met is expressed in vascular endothelial cells and is upregulated along with HGF in response to environmental stress $(18,19)$. The results of the present study show that c-Met 

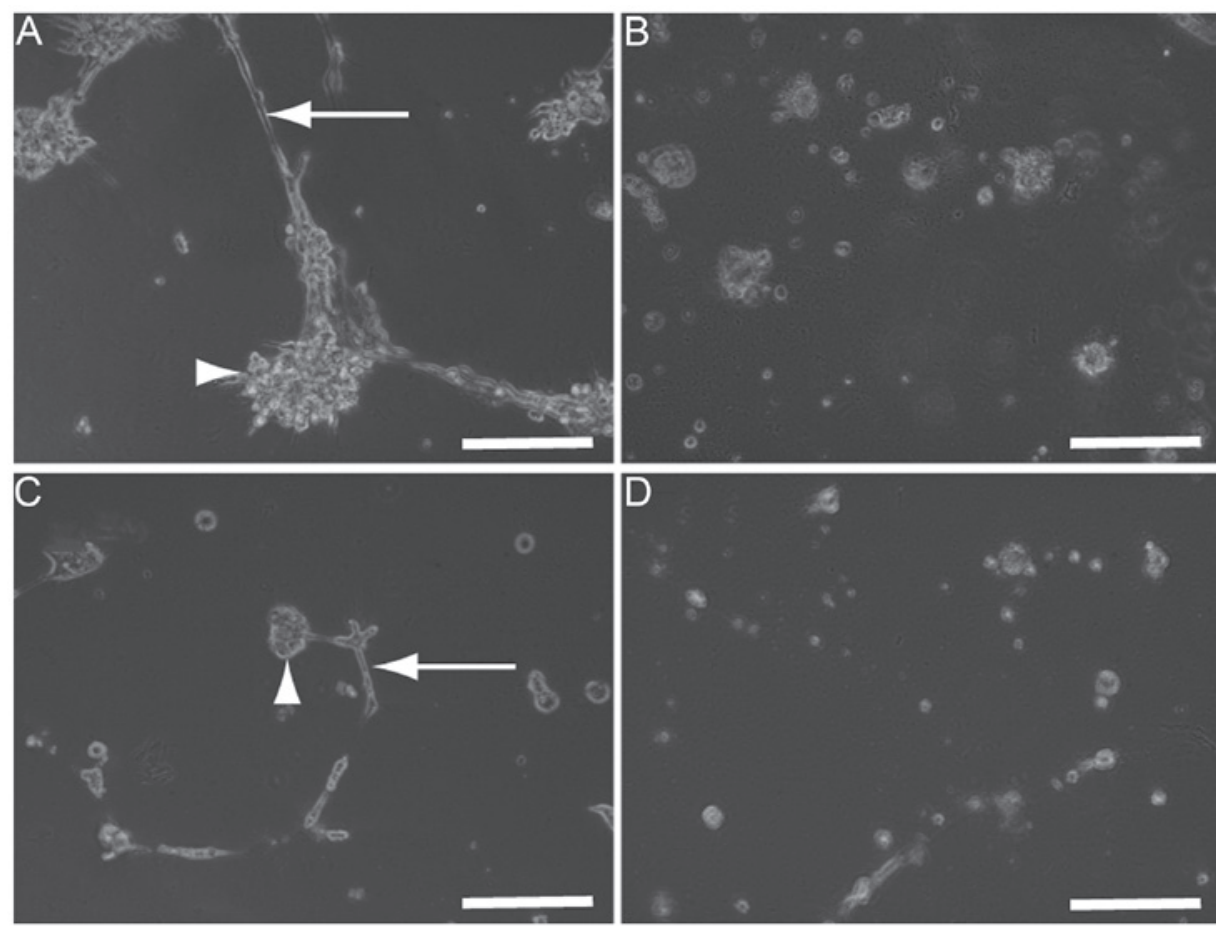

Figure 6. Co-culture of hepatocellular carcinoma cells and human umbilical vein endothelial cells (HUVECs). As a model of hepatoma cells and vascular vessels in hepatoma tissues, co-cultures of (A) HLF cells or (B) PLC/PRL/5 cells with (C) HUVECs were established. Three days after the addition of $30 \mu \mathrm{M}$ SU11274, the co-culture of HUVECs with (B) HLF or (D) PLC/PRL/5 cells were damaged. Arrow, HUVECs; arrowhead, hepatocellular cancer cells; original magnification, $\mathrm{x} 100$; scale bar, $50 \mu \mathrm{m}$.

is expressed in HUVECs and the proliferation of HUVECs is suppressed by SU11274. This indicates that SU11274 may suppress the proliferation of HCC cells and HUVECs. Co-cultures of HLF or PLC/PRL/5 cells and HUVECs were established to assess the anti-proliferative effects of SU11274. The data clearly show that these co-cultures were damaged by SU11274 treatment, indicating that SU11274 may be useful for the suppression of proliferation of HCC cells, as well as angiogenesis.

In conclusion, except for Hep3B, c-Met is highly expressed in hepatoma cells and HUVECs. SU11274 (30 $\mu \mathrm{M})$ suppresses the proliferation of HLF, PLC/PRL/5 and HUVECs and it was found that $30 \mu \mathrm{M}$ SU11274 damaged the co-culture of HLF or PLC/PRL/5 cells with HUVECs.

\section{References}

1. Lencioni R, Petruzzi P and Crocetti L: Chemoembolization of hepatocellular carcinoma. Semin Intervent Radiol 30: 3-11, 2013.

2. Kim HY and Park JW: Clinical trials of combined molecular targeted therapy and locoregional therapy in hepatocellular carcinoma: past, present, and future. Liver Cancer 3: 9-17, 2014.

3. Tomizawa M, Kondo F and Kondo Y: Growth patterns and interstitial invasion of small hepatocellular carcinoma. Pathol Int 45: 352-358, 1995.

4. Miyahara K, Nouso K, Morimoto Y, et al; Okayama Liver Cancer Group: Pro-angiogenic cytokines for prediction of outcomes in patients with advanced hepatocellular carcinoma. Br J Cancer 109: 2072-2078, 2013.

5. Xie B, Wang DH and Spechler SJ: Sorafenib for treatment of hepatocellular carcinoma: a systematic review. Dig Dis Sci 57: 1122-1129, 2012.

6. Furuse J, Ishii H, Nakachi K, Suzuki E, Shimizu S and Nakajima K: Phase I study of sorafenib in Japanese patients with hepatocellular carcinoma. Cancer Sci 99: 159-165, 2008.
7. Hu H, Duan Z, Long X, et al: Sorafenib combined with transarterial chemoembolization versus transarterial chemoembolization alone for advanced-stage hepatocellular carcinoma: a propensity score matching study. PLoS One 9: e96620, 2014.

8. Nishikawa H, Takeda H, Tsuchiya K, et al; Japanese Red Cross Liver Study Group: Sorafenib therapy for BCLC stage B/C hepatocellular carcinoma; clinical outcome and safety in aged patients: a multicenter study in Japan. J Cancer 5: 499-509, 2014.

9. Organ SL and Tsao MS: An overview of the c-MET signaling pathway. Ther Adv Med Oncol 3 (Suppl 1): S7-S19, 2011.

10. Grigioni WF, Fiorentino M, D'Errico A, et al: Overexpression of c-met protooncogene product and raised Ki67 index in hepatocellular carcinomas with respect to benign liver conditions. Hepatology 21: 1543-1546, 1995.

11. Kang GH, Lee BS, Lee ES, Kim SH, Lee HY and Kang DY: Prognostic significance of p53, mTOR, c-Met, IGF-1R, and HSP70 overexpression after the resection of hepatocellular carcinoma. Gut Liver 8: 79-87, 2014.

12. Scagliotti GV, Novello $S$ and von Pawel J: The emerging role of MET/HGF inhibitors in oncology. Cancer Treat Rev 39: 793-801, 2013.

13. Mughal A, Aslam HM, Sheikh A, Khan AM and Saleem S: c-Met inhibitors. Infect Agent Cancer 8: 13, 2013.

14. Inagaki Y, Qi F, Gao J, et al: Effect of c-Met inhibitor SU11274 on hepatocellular carcinoma cell growth. Biosci Trends 5: 52-56, 2011.

15. Dang Y, Luo D, Rong M and Chen G: Underexpression of miR-34a in hepatocellular carcinoma and its contribution towards enhancement of proliferating inhibitory effects of agents targeting c-MET. PLoS One 8: e61054, 2013.

16. Tomizawa M, Shinozaki F, Sugiyama T, Yamamoto S, Sueishi M and Yoshida T: Insulin-like growth factor I receptor involvement in proliferation of NOR-P1 cells in serum-free media. J Cell Biochem 113: 2714-2720, 2012.

17. Tomizawa M, Shinozaki F, Motoyoshi Y, et al: Niclosamide suppresses hepatoma cell proliferation via the Wnt pathway. Onco Targets Ther 6: 1685-1693, 2013.

18. Harrington LS, Sainson RC, Williams CK, et al: Regulation of multiple angiogenic pathways by DII4 and Notch in human umbilical vein endothelial cells. Microvasc Res 75: 144-154, 2008.

19. Hu SY, Duan HF, Li QF, et al: Hepatocyte growth factor protects endothelial cells against gamma ray irradiation-induced damage. Acta Pharmacol Sin 30: 1415-1420, 2009. 